\title{
Validación del Cuestionario Tridimensional para la Depresión (CTD) en población venezolana con enfermedades crónicas
}

\author{
Irama Cardozo ${ }^{1}$, Leticia Guarino ${ }^{2}$ y José Eduardo Rondón ${ }^{1}$ \\ ${ }^{1}$ Universidad Central de Venezuela, Caracas, Venezuela. \\ ${ }^{2}$ Universidad Simón Bolivar, Caracas, Venezuela.
}

Resumen: Esta investigación tuvo como objetivo validar el Cuestionario Tridimensional para la Depresión (Jiménez y Miguel-Tobal 2003) en población venezolana con enfermedades crónicas. Se evaluó la estructura factorial a través de un análisis de componentes principales, así como su consistencia interna y sus asociaciones con el inventario de Ansiedad Rasgo de Spielberg en una muestra de 202 pacientes con diabetes mellitus, hipertensión y enfermedades ano-rectales. Se obtuvo una fiabilidad aceptable $(\alpha=.857)$ y una estructura de tres componentes (motor, cognitivo y fisiológico). La correlación con la dimensión de ansiedad rasgo fue satisfactoria $(r=.706)$, lo que sugiere que el cuestionario posee adecuada validez convergente. Posteriormente, se realizó un análisis factorial confirmatorio con una muestra diferente de 322 pacientes, cuyos resultados demuestran un mal ajuste de los datos. Se recomienda verificar los resultados, analizando las propiedades psicométricas del cuestionario con otras muestras de personas con enfermedades crónicas.

Palabras clave: Depresión; Cuestionario Tridimensional para la Depresión; evaluación psicológica; enfermedades crónicas.

Tridimensional Depression Questionnaire: Validation in a Venezuelan population with chronic diseases

\begin{abstract}
The aim of the present study was to validate the Tridimensional Depression Questionnaire (Jiménez y Miguel-Tobal, 2003) in a Venezuelan population with chronic diseases. The factorial structure was analyzed using a principal component analysis (PCA), as well as the internal consistency and correlations with Spielberg's State-Trait Anxiety Inventory, in a sample of 202 patients with diabetes mellitus, hypertension, and ano-rectal diseases. An adequate reliability $(\alpha=.857)$ and a three factor structure (motor, cognitive and physiological) were obtained. The correlation with trait anxiety was satisfactory $(r=.706)$, which indicates an adequate convergent validity of the questionnaire. A confirmatory factor analysis (CFA) was then conducted with a different sample of 322 patients, with bad adjustment results. It is recommended to verify the results, analyzing the questionnaire's psychometric properties with other samples of patients with chronic diseases.
\end{abstract}

Keywords: Depression; Tridimensional Depression Questionnaire; psychological assessment; chronic diseases.

\section{Introducción}

La depresión, uno de los trastornos más frecuentes en las diferentes etapas del ciclo vital, modifica negativamente la funcionalidad del individuo en su entorno y

\section{Recibido: 3 octubre 2015; aceptado: 12 febrero 2016.}

Correspondencia: Irama Cardozo Quintana, Instituto de Psicología, Universidad Central de Venezuela, Avenida Neverí, Centro Comercial Los Chaguaramos, Piso 2. Oficina 2-11. Urbanización Los Chaguaramos, 1040 Parroquia San Pedro, Caracas, Distrito Capital, Venezuela. Correo-e: irama.cardozo@ucv.ve puede incrementar, entre otras cosas, la tendencia al suicidio y al consumo de sustancias, así como la probabilidad de desarrollar enfermedades (Cano-Vindel, Salguero, Wood, Dongil y Latorre, 2012; Gutiérrez y Portillo, 2013; Wagner, González-Fortaleza, Sánchez-García, García-Peña y Gallo, 2012).

Se han planteado distintos modelos explicativos para este trastorno, entre los que se encuentran los biológicos, cognitivos, de autocontrol, de déficit en las estrategias de afrontamiento, o los de solución de problemas (Gotlib y Joormann, 2010; Jiménez y Miguel-Tobal, 2003). Igualmente, se plantea que la depresión debe es- 
tudiarse en tres niveles: sintomatológico, sindrómico y como trastorno clínico (Vázquez, Hernangómez, Hervás y Nieto-Moreno, 2006).El nivel sintomatológico alude a un declive en el estado de ánimo, lo que se traduce en tristeza y desánimo. Por su parte, el nivel sindrómico implica además de la tristeza, síntomas concomitantes tales como cambios en los hábitos de alimentación, sueño, higiene, cogniciones pesimistas y evaluación negativa del entorno. La depresión como trastorno clínico, alude a aquella psicopatología que no se debe a la existencia de un evento estresante que la haya causado y permanece durante un tiempo prolongado. En función del nivel en el que se esté ubicado, la evaluación y el abordaje son distintos (Vázquez et. al., 2006).

La depresión como síndrome, implica un estado de ánimo deprimido (tristeza, abatimiento) y otros síntomas fisiológicos, cognitivos y motores (Gotlib y Joormann, 2010; Piqueras, Martínez, Ramos, Rivero y García, 2006; Piqueras, Martínez, Ramos, Rivero, García y Oblitas, 2008; Jiménez y Miguel-Tobal, 2003), como los siguientes: Síntomas fisiológicos: falta de apetito, alteraciones del sueño (pérdida o aumento), reducción del deseo sexual, y molestias somáticas. Estas manifestaciones tienen que ver con la activación del eje hipotalámico-hipofisiario-suprarrenal, el descontrol en la neurotransmisión entre los hemisferios cerebrales y el sistema límbico, específicamente el desajuste de la noradrenalina, serotonina, acetilcolina y las endorfinas, lo que conlleva una menor secreción de cortisol y de las hormonas tiroideas y del crecimiento. Sintomas cognitivos: pérdida de interés por las actividades, desánimo, desesperanza, inseguridad pérdida de autoestima, percepción negativa del entorno, sentimientos de culpa, déficits de memoria, dificultad para la concentración, auto-depreciación, sensación de lentitud, dificultad para tomar decisiones, percepción de fracaso, pensamientos suicidas, entre otros. Sintomas motores: rechazo a realizar las actividades cotidianas, sean laborales, recreativas o de otra índole, descuido en el aseo personal, exceso de inactividad, poca espontaneidad en las expresiones, voz pausada y con entonación pobre, retardo psicomotor o por el contrario agitación motora (movimiento excesivo de manos o pies, morderse las uñas, frotar manos).

Existen diversas escalas para evaluar la depresión en población general, así como en grupos de pacientes con enfermedades, entre las que se encuentran el Inventario para la Depresión de Beck (BDI) cuya versión más actualizada (BDI-II) ha sido validada y empleada tanto en población general como clínica (Moral, 2013; Sanz y García-Vera, 2013), la escala de Zung para de Depresión (Cogollo, Díaz y Campo, 2006; Ruiz-Grosso et al., 2012; Trento et al., 2012) y la Escala Hospitalaria de Ansiedad y Depresión (HADS) que permite evaluar la ansiedad y la depresión en pacientes con algún tipo de enfermedad y cuya eficacia se ha determinado en distintas culturas y con personas con distintas patologías tales como el cáncer y el Virus de Inmunodeficiencia Humana (Nogueda, Pérez, Barrientos, Robles y Sierra, 2013).

Pese a que las escalas antes mencionadas se han empleado en distintas culturas y con distintas poblaciones y gozan de validez y confiabilidad para la evaluación de la depresión, Jiménez y Miguel-Tobal (2003), basados en el modelo tridimensional de las emociones descrito por Lang (1968),propusieron el Cuestionario Tridimensional para la Depresión (CTD), un instrumento para evaluar la depresión en sus tres dimensiones: cognitiva, fisiológica y conductual/motor (Martínez-Monteagudo, Inglés, Cano-Vindel y García-Fernández, 2012). De acuerdo con el modelo tridimensional, las emociones se manifiestan según un triple sistema de respuesta donde cada dimensión tiene características particulares y puede variar independientemente de los cambios de las otras dimensiones. Los tres componentes se manifiestan de la manera como se explicó anteriormente en el caso particular de la depresión: cognitivo, que implica pensamientos e imágenes relacionadas con la situación; fisiológico, relacionado con incremento de la actividad del Sistema Nervioso Autónomo, el Sistema Nervioso Somático e incremento de la actividad neuroendocrina del Sistema Nervioso Central; motor, que involucra respuestas directas como inquietud motora, tartamudeo, gesticulaciones particulares y respuestas indirectas, que refieren a los comportamientos involuntarios de escape y evitación de la situación(Martínez-Monteagudo et al., 2012). Considerando las manifestaciones de la depresión en los aspectos indicados, es preciso contar con instrumentos que evalúen los tres componentes, con la finalidad de que al emplearlos, se obtenga información acerca de la gravedad de la mayor parte de los indicadores de depresión y de esta manera derivar estrategias de abordaje pertinentes.

En este sentido, el CTD (Jiménez y Miguel-Tobal, 2003) ofrece una medida de la depresión basada en estas tres dimensiones, una puntuación total para medir la gravedad de la depresión y la tendencia suicida de la persona evaluada. Está formado por cuatro escalas: cognitiva, fisiológica, motora y tendencia suicida. Cabe señalar que el instrumento incluye esta última escala debido a que tras el análisis factorial llevado a cabo para la construcción de la versión definitiva, se encontró que la solución que explicaba una mayor porcentaje de varianza estaba constituida por cuatro factores: los tres que se adaptan al modelo teórico de los tres sistemas de respuesta y un cuarto en el que se agruparon todos los ítems que hacían referencia a la tendencia suicida. 
Debido a que este instrumento permite evaluar la depresión considerando ítems que involucran los tres sistemas de respuesta y demostrada su validez y consistencia interna, se considera que podría ser un instrumento valioso para evaluar la depresión no solo en población general, sino en personas con enfermedades médicas crónicas, pues esta problemática psicológica es una de las más prevalentes en las personas que presentan este tipo de enfermedades, siendo entendida como una causa de distintas patologías y a la vez una consecuencia de los síntomas, lo cual afecta a su vez la respuesta a los tratamientos aplicados y el incremento de la percepción de síntomas (Edo y Ballester, 2006; Gil, Ballester, Gómez y Abizanda, 2013; Gros, Anthony, McCabe y Swinson, 2009; Iwamitsu, et al., 2003); Oblitas, 2010; Pescatori, Spyrou y Pulvirenti d'Urso, 2006; Piqueras et al., 2006; Stenman, Holzmann y Sartipy, 2014). Por su parte, Fernández y Miralles (2014) estudiaron la depresión en relación con el asma bronquial empleando el CTD, y Salguero e Iruarrizaga (2006) estudiaron la relación entre la inteligencia emocional percibida y la emocionalidad negativa empleando el CTD como cuestionario para evaluar la depresión.

Cano-Vindel y Tobal (2001, cp. Piqueras etal., 2006) plantean que la relación depresión - enfermedad se explica de tres maneras: a)Si la depresión se mantiene en el tiempo, puede producir cambios conductuales permanentes que son incompatibles con los hábitos necesarios para mantener la salud; b) los niveles de activación biológica que se suscitan influyen negativamente en la salud; y c) la alta activación biológica puede generar una inmunosupresión, que se traduce en mayor vulnerabilidad a las enfermedades. En este sentido, hallazgos de diferentes investigaciones (Bharucha, y Wald, 2010; Frasure-Smith, Lesperance y Talajic, 1995; Piqueras et al., 2008; Redondo, Calvanesse, García y Hernández, 2010; Stenman et al., 2014) han mostrado la relación existente entre la depresión y la presencia de cardiopatías, nefropatías, problemas gastrointestinales y reumáticos, entre otros. Asimismo, altos niveles de depresión, la presencia de síntomas depresivos, la distimia y la historia de depresión mayor, permiten predecir enfermedades cardiovasculares, eventos cardíacos y un alto riesgo de desarrollar diabetes. De hecho, en estudios longitudinales se ha encontrado que las personas con altos niveles de depresión presentan una doble probabilidad de haber muerto de cáncer, en comparación con aquellas que no presentan depresión (Shekelle 1981; cp. Piqueras et al., 2008).

Debido a la gran prevalencia de la depresión en las personas que padecen enfermedades médicas crónicas, es importante contar con instrumentos válidos y confia- bles que permitan evaluar este trastorno en dicha población. En este sentido, el objetivo del presente trabajo es realizar la validación del Cuestionario Tridimensional para la Depresión (CTD) en población venezolana con enfermedades médicas crónicas.

\section{Método}

\section{Participantes}

Para el desarrollo del presente estudio se emplearon dos muestras de personas: una para la realización del análisis factorial exploratorio (AFE) y análisis de las características psicométricas iniciales del cuestionario, $\mathrm{y}$ una segunda para la realización del análisis factorial confirmatorio (AFC).

(a) Muestra 1. Conformada por 202 venezolanos adultos (157 mujeres, 77.7\%), con edades comprendidas entre los 18 y 75 años $(M=56.30, D T=12.57)$, que asistían al Hospital Universitario de Caracas y a la Fundación Antidiabética por presentar las siguientes enfermedades crónicas: diabetes mellitus tipo 1 y $2(n=101$; $50 \%)$, hipertensión $(n=60 ; 29.70 \%)$ y trastornos ano-rectales $(n=41 ; 20.29 \%)$.

(b) Muestra 2. Conformada por 322 participantes (241 mujeres, $74.8 \%$ ), con edades comprendidas entre los 17 y 81 años $(M=53, D T=13.89)$, que asistían al Hospital Universitario de Caracas y a la Fundación Antidiabética por presentar las siguientes enfermedades crónicas: diabetes mellitus tipo 1 y $2(n=101 ; 31.4 \%)$, hipertensión $(n=60 ; 18.6 \%)$ y trastornos ano-rectales $(n=161 ; 50 \%)$.Esta muestra completó un cuestionario compuesto por 32 ítems, pues se retiraron aquellos que quedaron excluidos tras el AFE realizado con la primera muestra (ítems 11 y 24).

\section{Instrumentos}

Hoja de identificación: Consta de una serie de preguntas sobre datos personales de los pacientes: sexo, edad, patología, tipo de diabetes, tiempo desde el primer diagnóstico, y presencia de otras enfermedades.

Cuestionario Tridimensional para la Depresión (CTD; Jiménez y Miguel-Tobal, 2003):constituido por 34 ítems que evalúan tres sistemas de respuesta: cognitivo (10 ítems), fisiológico (10 ítems) y motor ( 7 ítems). Además 7ítems evalúan tendencia suicida. La escala de respuesta va de 0 (casi nunca) a 4 (casi siempre). El rango de puntajes para el cuestionario total oscila entre 0 y 136 , y una puntuación alta indica un mayor nivel de depresión. De acuerdo con los autores, los índices de fiabilidad test-retest en todas las escalas son superiores a.60, mien- 
tras que el índice de consistencia interna de la prueba total es de.96, y el de cada componente .92 (cognitiva), .90 (fisiológica), .88 (motora) y .91 (tendencia suicida). La validez convergente oscila entre. 41 y. 89 y presenta una estructura factorial que se corresponde con la planteada teóricamente, explicando un $61.41 \%$ de la varianza total.

Inventario de Ansiedad Rasgo (STAI-R). Se trata de la sub-escala del Inventario de Ansiedad Estado/Rasgo de Spielberger, Gorsuch y Lushene (1982), que evalúa el rasgo de ansiedad a través de 20 reactivos. Cada ítem tiene cuatro opciones de respuesta de 1 (casi nunca) a 4 (casi siempre). Las puntuaciones varían en un rango del 20 al 80, y una puntuación alta indica una mayor tendencia a responder de manera ansiosa ante situaciones demandantes (rasgo). La validación española más reciente (Teva, Bermúdez, Hernández-Quero y Buela-Casal, 2005) muestra una consistencia interna de $\alpha=.82$.

\section{Procedimiento}

Para la selección de los participantes se empleó un tipo de muestro no probabilístico de tipo propositivo (Kerlinger y Lee, 2002). Los pacientes debían presentar las siguientes características: (a) Diagnóstico médico de diabetes mellitus tipo 1 y 2 (mínimo 1 año con el diagnóstico), hipertensión arterial o patologías ano-rectales tales como estreñimiento crónico, enfermedad hemorroidal, fisura anal, entre otras, no asociadas a enfermedad o malformación congénita; (b) Los pacientes con diabetes, no debían presentar comorbilidad con retinopatía, nefropatía ni amputaciones. Por su parte, los pacientes con trastornos ano-rectales no debían tener trastornos concomitantes como VPH anal, fístulas sifilíticas, cáncer de colon, enfermedad de Crohn, recto-colitis ulcerosa o síndrome de intestino irritable; (c) Saber leer y escribir; (d) No encontrarse hospitalizado ni en situación de post-operatorio; (e) No presentar ninguna patología psiquiátrica diagnosticada.

Se solicitó el permiso a las autoridades de la Fundación Antidiabética, Hospital Universitario de Caracas, ambas instituciones ubicadas en Caracas - Venezuela, para utilizar sus instalaciones y entrevistar a sus pacientes. Una vez seleccionados los participantes según los criterios establecidos, se les indicó el propósito de la investigación y se solicitó por escrito su consentimiento de participación, cumpliéndose de esta manera con los parámetros éticos de la investigación. Luego se aplicaron los cuestionarios en forma de entrevista individualizada.

\section{Análisis estadísticos}

Completados los datos de la muestra 1 , se procedió a su codificación, vaciado y procesamiento a través del pa- quete estadístico SPSS versión 20, para realizar el análisis de componentes principales. Previamente se determinó si era posible realizar el análisis factorial a través de la prueba de adecuación muestral de Kaiser-Meyer-Olkin y el test de esfericidad de Bartlett y luego se efectuó el análisis factorial de componentes principales con rotación Varimax.

Posteriormente, con la muestra 2 se procedió a realizar un análisis factorial confirmatorio a través del paquete estadístico AMOS. Previamente se evaluó la adecuación de la muestra (KMO) y la prueba de esfericidad de Bartlett para evaluar los supuestos relacionados con la matriz de correlación. Finalmente, se evaluó el modelo con el método de Máxima Verosimilitud. Se evaluaron los índices de ajuste del modelo: Chi cuadrado, índice de ajuste comparativo (CFI),índice Tucker-Lewis (TLI),índice de ajuste normado (NFI) y error de aproximación cuadrático medio (RMSEA) (Hair, Anderson, Tatham y Black, 2008).

\section{Resultados}

\section{Resultados preliminares}

Al analizar los ítems se observa un promedio en cada ítem alrededor de 1 a 2 puntos, es decir, en las categorías a veces y a menudo, evidenciándose que la mayoría de los pacientes presentó un bajo nivel de depresión. Los puntajes más bajos se observaron en dos de los ítems relacionados con las ideas suicidas $(18,6)$ así como en el ítem 14. Los ítems con medias más altas son el 3, 4 y 5. Asimismo, la respuesta a los ítems es heterogénea (ver Tabla 1).

\section{Análisis factorial exploratorio y consistencia interna}

Con la finalidad de explorar desde el punto de vista estadístico la pertinencia de llevar a cabo el AFE, se realizaron los test de adecuación muestral de Kaiser-Meyer-Olkin, que arrojó un índice de .765 , que indica que es apropiado aplicar el análisis, pues se encuentra entre 0.5 y 1 , tal como lo señala Montoya (2007). Así mismo, se aplicó el test de esfericidad de Bartlett, para probar la hipótesis nula de que las variables no están correlacionadas en la población, es decir, si la matriz de correlaciones es una matriz de identidad (Montoya, 2007). En este caso, es significativa $(p<.001)$, por lo que se rechaza la hipótesis nula y se considera que se puede continuar con el análisis factorial.

Con el criterio de valores propios cercanos y superiores a 1.9 se obtuvieron tres factores que explicaron el $31.94 \%$ de la varianza total. El primer factor, con un valor propio de 6.36, explicó el $18.98 \%$ de la varianza, el 
Tabla 1 .Estadísticos descriptivos $(M$ y $D T)$ para cada uno de los ítems del Cuestionario Tridimensional para la Depresión

\begin{tabular}{|c|c|c|}
\hline Ítem & Media & $D T$ \\
\hline $\begin{array}{l}\text { 1. Mis actividades placenteras y de ocio se } \\
\text { han reducido }\end{array}$ & 1.62 & 1.60 \\
\hline $\begin{array}{l}\text { 2. Pienso que es mejor que estuviera muer- } \\
\text { to/a }\end{array}$ & 0.31 & 0.86 \\
\hline 3. Tengo dolores musculares & 2.06 & 1.54 \\
\hline $\begin{array}{l}\text { 4. Me despierto con facilidad durante la no- } \\
\text { che }\end{array}$ & 2.16 & 1.68 \\
\hline 5. Me preocupo con facilidad & 2.54 & 1.60 \\
\hline 6. Pienso en el suicidio & 0.08 & 0.47 \\
\hline 7. Mis movimientos son lentos & 1.09 & 1.46 \\
\hline 8. Me duelen las articulaciones & 1.96 & 1.61 \\
\hline 9. Tengo insomnio & 1.38 & 1.61 \\
\hline 10. Me siento a disgusto con mi aspecto físico & 0.61 & 1.19 \\
\hline 11. En casa me encierro en mi habitación & 0.80 & 1.38 \\
\hline $\begin{array}{l}\text { 12. Cuando estoy en un grupo de gente me } \\
\text { siento inferior }\end{array}$ & 0.16 & 0.66 \\
\hline $\begin{array}{l}\text { 13. Tiendo a sentirme culpable si las cosas no } \\
\text { van bien }\end{array}$ & 0.61 & 1.01 \\
\hline 14. Creo que los demás valen más que yo & 0.08 & 0.45 \\
\hline 15. Hago menos cosas de las que hacía & 1.58 & 1.56 \\
\hline $\begin{array}{l}\text { 16. Creo haber decepcionado a las personas } \\
\text { que me quieren }\end{array}$ & 0.46 & 1.03 \\
\hline 17. Tengo molestias en el estómago & 1.33 & 1.61 \\
\hline 18. Siento deseos de acabar con mi vida & 0.07 & 0.38 \\
\hline $\begin{array}{l}\text { 19. Siento una gran desesperación, como si es- } \\
\text { tuviese en una situación sin salida }\end{array}$ & 0.63 & 1.00 \\
\hline 20. Mis relaciones sexuales han disminuido & 1.78 & 1.65 \\
\hline 21. Tengo dificultades para relajarme & 1.03 & 1.43 \\
\hline 22. Pienso que soy una carga para los demás & 0.26 & 0.79 \\
\hline 23. Me asusta pensar en el futuro & 0.71 & 1.21 \\
\hline 24. Pienso que todo me va a salir mal & 0.24 & 0.73 \\
\hline 25. Llamo menos que antes a mis amigos & 1.23 & 1.53 \\
\hline $\begin{array}{l}\text { 26. El corazón me late muy rápido y noto pal- } \\
\text { pitaciones }\end{array}$ & 1.02 & 1.34 \\
\hline 27. Me cuesta mucho tomar decisiones & 0.88 & 1.29 \\
\hline $\begin{array}{l}\text { 28. Tengo problemas para concentrarme y } \\
\text { pensar con claridad }\end{array}$ & 0.83 & 1.30 \\
\hline 29. Descuido mi aspecto físico & 0.63 & 1.12 \\
\hline 30. Salgo menos que antes & 1.72 & 1.59 \\
\hline 31. Mi respiración se agita & 0.98 & 1.26 \\
\hline $\begin{array}{l}\text { 32. Me siento físicamente mal, con molestias } \\
\text { corporales }\end{array}$ & 1.39 & 1.52 \\
\hline 33. Me arreglo menos & 1.01 & 1.47 \\
\hline 34. Lloro de forma incontrolada & 0.55 & 1.06 \\
\hline
\end{tabular}

segundo con un valor propio de 2.56 explicó el 7.52\%, mientras que el tercer factor arrojó un valor propio de 1.93 y explicó el $5.68 \%$ de la varianza total. En el gráfico de sedimentación se observó que hasta el codo de la curva había tres factores y al examinar las cargas factoriales (igual o mayor a .28), todos los ítems saturaban en los tres factores propuestos, excepto los ítems 11 y 24 .
En la tabla de componentes rotados (Tabla 2), se muestra la estructura factorial del cuestionario, observándose que empleando un criterio de saturación $\geq 0.28$ en el factor 1 se incluyen 7 ítems que, según los autores y de acuerdo con su contenido, se corresponden con el componente motor de la depresión. Además en este factor se incluyeron los ítems 20,31, 27 y 28, que hacen referencia a problemas cognitivos $(27$ y 28$)$ y a dificultades físicas $(20,31)$.

El factor 2 está formado por 6 ítems que, según los autores del cuestionario, forman parte del componente cognitivo de la depresión. Así mismo, en este factor cargaron los ítems 2, 6, 18, 19, y 22 que inicialmente pertenecen a la escala de ideación suicida. Asimismo, se incluyó en este factor el ítem 21quepertenece al componente fisiológico. Finalmente, el factor 3agrupa 8 ítems que por sus características se corresponden con el componente fisiológico de la depresión, tal como lo indican los autores, además del ítem 34 que, según la teoría, pertenece a la escala de ideación suicida. Por su parte, los ítems 11 "En casa me encierro en mi habitación" y 24 "Pienso que todo me va a salir mal", quedaron excluidos del cuestionario, debido a que no presentaron saturaciones significativas en ningún factor.

Debido a que la estructura encontrada excluyó dos ítems y explicó un porcentaje de varianza menor al encontrado con la versión original del instrumento, se realizó un análisis en el que se forzó la estructura de 4 factores, que mostró un porcentaje de varianza ligeramente mayor (36.81\%). Sin embargo, se observó que en esta estructura 7 ítems presentaban solapamientos, con cargas factoriales muy similares, y los ítems que componen los 4 factores no se correspondían con claridad con la propuesta de los autores, además de excluirse el ítem 11.

En cuanto al análisis de consistencia interna de los componentes de la prueba, se observaron coeficientes moderadamente altos: Motor: $\alpha=.75$, Cognitivo: $\alpha=.75$, fisiológico: $\alpha=.76$, Total: $\alpha=.85$. Las correlaciones entre los tres factores fueron significativas $(p<.01)$ y moderadas, con valores que oscilaron entre .49 y .37 , mientras que las correlaciones de cada factor con la puntuación total fueron elevadas $(.81, .70, \mathrm{y} .81$, para los factores 1 , 2 , y 3 respectivamente).

\section{Validez convergente}

Por lo que se refiere a la validez convergente con la ansiedad rasgo, los resultados indicaron una correlación elevada $(r=.70, p<.01)$ entre las puntuaciones totales de ambos cuestionarios, lo que indica que las personas con altos niveles de depresión, presentan también alta tendencia a la ansiedad. Análogamente, se observaron 
Tabla 2. Análisis de componentes principales, con indicación de la matriz factorial rotada, del Cuestionario Tridimensional para la Depresión (CTD)

\begin{tabular}{|c|c|c|c|}
\hline \multirow[t]{2}{*}{ Ítems } & \multicolumn{3}{|c|}{ Componente } \\
\hline & 1 & 2 & 3 \\
\hline 27. Me cuesta mucho tomar decisiones & .65 & & \\
\hline $\begin{array}{l}\text { 28. Tengo problemas para concentrarme y } \\
\text { pensar con claridad }\end{array}$ & .54 & & \\
\hline 30. Salgo menos que antes & .54 & & \\
\hline 33. Me arreglo menos & .52 & & \\
\hline 15. Hago menos cosas de las que hacía & .50 & & \\
\hline 7. Mis movimientos son lentos & .50 & & \\
\hline 29. Descuido mi aspecto físico & .46 & & \\
\hline 20. Mis relaciones sexuales han disminuido & .45 & & \\
\hline 25. Llamo menos que antes a mis amigos & .44 & & \\
\hline $\begin{array}{l}\text { 1. Mis actividades placenteras y de ocio se } \\
\text { han reducido }\end{array}$ & .41 & & \\
\hline 31. Mi respiración se agita & .35 & & \\
\hline $\begin{array}{l}\text { 19. Siento una gran desesperación, como si } \\
\text { estuviese en una situación sin salida }\end{array}$ & & .62 & \\
\hline 22. Pienso que soy una carga para los demás & & .61 & \\
\hline 18. Siento deseos de acabar con mi vida & & .59 & \\
\hline 14. Creo que los demás valen más que yo & & .59 & \\
\hline $\begin{array}{l}\text { 16. Creo haber decepcionado a las personas } \\
\text { que me quieren }\end{array}$ & & .58 & \\
\hline 23. Me asusta pensar en el futuro & & .55 & \\
\hline $\begin{array}{l}\text { 10. Me siento a disgusto con mi aspecto fí- } \\
\text { sico }\end{array}$ & & .48 & \\
\hline $\begin{array}{l}\text { 13. Tiendo a sentirme culpable si las cosas } \\
\text { no van bien }\end{array}$ & & .46 & \\
\hline 21. Tengo dificultades para relajarme & & .42 & \\
\hline $\begin{array}{l}\text { 2. Pienso que es mejor que estuviera muer- } \\
\text { to/a }\end{array}$ & & .41 & \\
\hline $\begin{array}{l}\text { 12. Cuando estoy en un grupo de gente me } \\
\text { siento inferior }\end{array}$ & & .40 & \\
\hline 6. Pienso en el suicidio & & .33 & \\
\hline 24. Pienso que todo me va a salir mal & & & \\
\hline 11. En casa me encierro en mi habitación & & & \\
\hline 3. Tengo dolores musculares & & & .69 \\
\hline $\begin{array}{l}\text { 4. Me despierto con facilidad durante la } \\
\text { noche }\end{array}$ & & & .67 \\
\hline 8. Me duelen las articulaciones & & & .65 \\
\hline 9. Tengo insomnio & & & .61 \\
\hline 5. Me preocupo con facilidad & & & .56 \\
\hline $\begin{array}{l}\text { 32. Me siento físicamente mal, con moles- } \\
\text { tias corporales }\end{array}$ & & & .46 \\
\hline $\begin{array}{l}\text { 26. El corazón me late muy rápido y noto } \\
\text { palpitaciones }\end{array}$ & & & .36 \\
\hline 17. Tengo molestias en el estómago & & & .34 \\
\hline 34. Lloro de forma incontrolada & & & .28 \\
\hline
\end{tabular}

correlaciones moderadas y significativas entre cada factor del CTD y la ansiedad rasgo: $r=.59, r=.66, r=$ .61 ,para los factores motor, cognitivo, y fisiológico, respectivamente.

\section{Análisis factorial confirmatorio}

Como se ha indicado anteriormente, este análisis e llevó a cabo con la muestra 2. En primer lugar se evaluó la prueba de adecuación de la muestra $(K M O=.84)$ y tras la aplicación de la prueba de esfericidad de Bartlett que resultó ser significativa $(X 2=3102.16 ;$ g.l. $=561 ; p<$ $.001)$, por lo que se cumplen los supuestos referentes a la matriz de correlación (Hair et.al., 2008), se procedió a realizar el análisis factorial.

Se evaluó el modelo de tres factores por el método de Máxima Verosimilitud (ver Figura 1) obteniéndose coeficientes estimados moderados entre cada ítem y el factor motor de entre .45 y .62, siendo el ítem con más carga el $\mathrm{n}^{\mathrm{o}} 7$ ("Mis movimientos son lentos") y el de menor carga el ítem 25 ("Llamo menos que antes a mis amigos"). Por otra parte, se encontraron coeficientes moderados-altos y bajos entre cada ítem y el factor cognitivo (entre $.18 \mathrm{y}$ .75), siendo el ítem con mayor carga el $n^{\circ} 19$ ("Siento una gran desesperación, como si estuviese en una situación sin salida") y el de menor el $n^{\circ} 6$ ("Pienso en el suicidio"). Finalmente se encontraron coeficientes moderados de entre .32 y .64 entre cada ítem y el componente fisiológico siendo el ítem con mayor carga el 32 ("Me siento físicamente mal, con molestias corporales") y el de menor carga el 17 ("Tengo molestias en el estómago").

Respecto a los índices de ajuste de este modelo se obtuvo un valor de $\mathrm{Chi}^{2}$ significativo con un nivel de confianza del 95\% $\left(X^{2}=1133.322 ;\right.$ g.l. $\left.=461 ; p<.001\right)$, lo que indica un mal ajuste. Dado que este índice es vulnerable al tamaño de la muestra se analizaron también otros índices, obteniéndose los valores siguientes: $C F I=.73 ; T L I=.71 ; N F I=.62 ; R M S E A=.06$. Dado que los primeros están muy por debajo de .90 , y el último se encuentra por debajo de .10 , cabe concluir que los datos presentan un mal ajuste (Hair et al., 2008).

Al igual que en el análisis factorial exploratorio, debido a que los autores del instrumento original propusieron una estructura de 4 factores, se realizó un análisis factorial confirmatorio con dicha estructura. Esta estructura mostró un mal ajuste tras analizar los distintos índices, que fueron menos adecuados que los arrojados por la estructura de 3 factores $(X 2=1336,707 g . l .=521$, $p<.001 ; C F I=.694 ; T L I=.67 ; N F I=.585 ;$ RMSEA $=.07$ ).

En cuanto a la consistencia interna, los valores obtenidos fueron los siguientes: factor motor: $\alpha=.79$; factor cognitivo: $\alpha=.75$; factor fisiológico: $\alpha=.78$; puntuación total: $\alpha=.88$. Las correlaciones entre los tres factores del cuestionario oscilaron entre $r=.44$ y $r=.59$. Las correlaciones entre los tres factores y la puntuación total fueron de $r=.87, r=.72$, y $r=.83$ para los factores mo- 


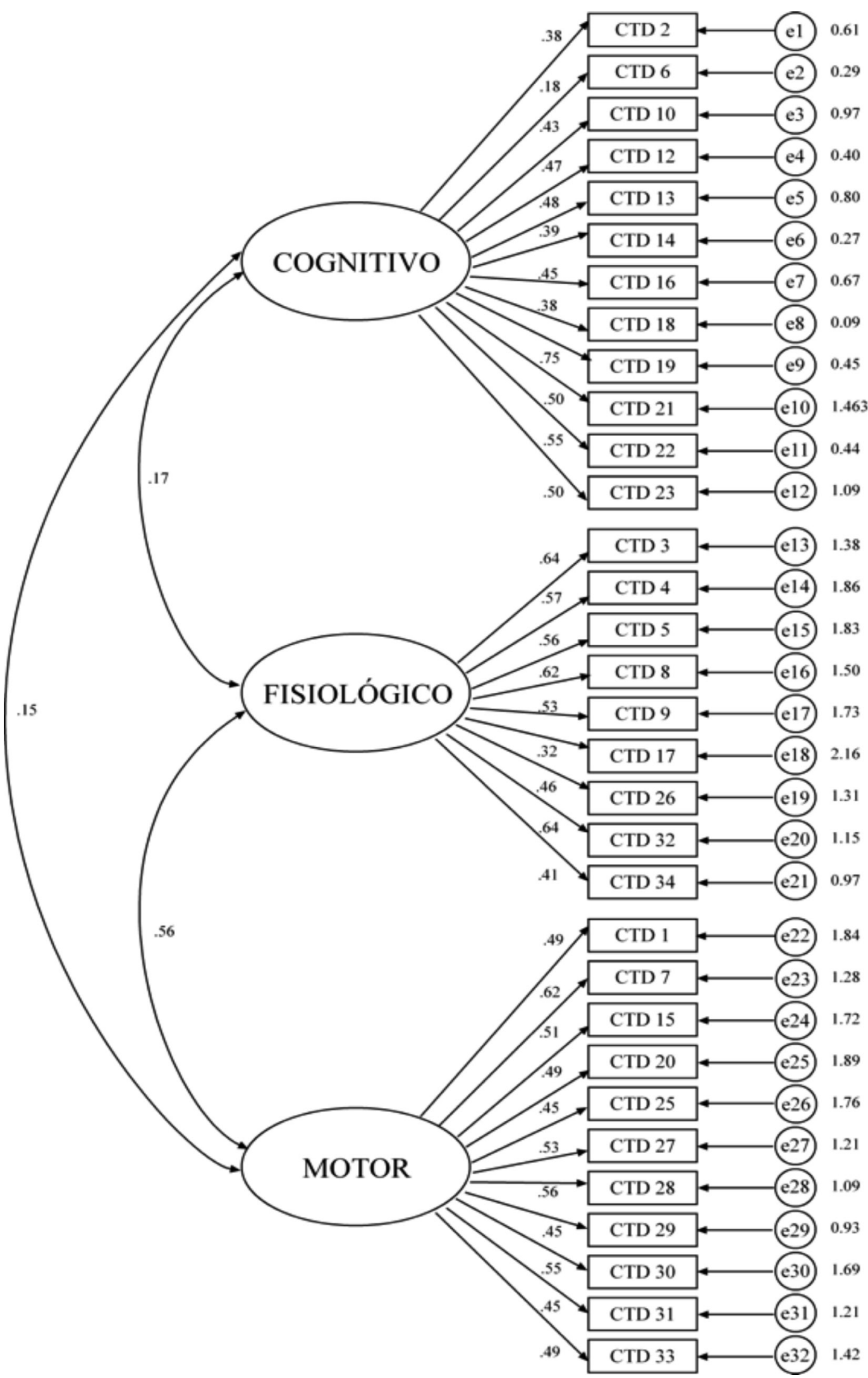

Figura 1. Análisis factorial confirmatorio del Cuestionario Tridimensional para la Depresión (CTD). Coeficientes estimados por ítem en el modelo de tres factores. 
tor, cognitivo y fisiológico, respectivamente. Todas estas correlaciones fueron significativas $(p \leq .01)$

\section{Discusión}

La presente investigación tuvo como objetivo realizar un estudio psicométrico del Cuestionario Tridimensional para la Depresión (CTD) en población venezolana con enfermedades crónicas, dada la relación que existe entre éstas y la depresión (Arechabala, Catoni, Palma y Barrios, 2011; Bounchoucha, Devroede y Arsac, 2004; Fabián, García y Cobo, 2010; Fernández, Simón y Bueno, 2010; Freire et al., 2011; Oladeju y Gureje 2013), lo que implica una necesidad de contar con instrumentos válidos y confiables que permitan evaluar la presencia de este trastorno en las personas que presentan problemas crónicos de salud.

El análisis relativo a la fiabilidad de la prueba muestra que presenta una alta consistencia interna, pues en el análisis con las dos muestras estudiadas se encontraron índices altos tanto en las tres dimensiones como en la puntuación total, lo que coincide con los hallazgos de Jiménez y Miguel-Tobal (2003) que encontraron índices superiores a. 83 .

En lo que refiere a los factores que componen la prueba, tras el análisis de componentes principales se encontró una estructura de tres factores (motor, cognitivo y fisiológico), que coincide con la propuesta inicial de los autores originales. No obstante, difiere de la que hallaron tras el análisis factorial, que indica que la prueba está constituida por cuatro factores(motor, cognitivo, fisiológico e ideación suicida)(Jiménez y Miguel-Tobal, 2003). La diferencia radica en que los autores además de las tres dimensiones que constituyen los niveles de respuesta motor, cognitivo y fisiológico, introducen la dimensión ideación suicida, mientras que en el presente trabajo los ítems de esta última escala $(2,6,18,19$ y 22$)$, excepto el ítem 34 ("Lloro de forma incontrolada") cargaron en el factor cognitivo, lo que puede estar explicado porque estos ítems aluden a pensamientos relacionados con la muerte, por lo que dichas ideas, independientemente de su contenido, forman parte del factor cognitivo.

Considerando que en el análisis factorial exploratorio la decisión del número de factores debe tener su sustento en la evidencia empírica (Pérez y Medrano, 2010) y tomando en cuenta que los factores encontrados en este estudio se corresponden con la propuesta original de los autores del instrumento y con el modelo teórico de base (Martínez-Monteagudo et al., 2012), es aceptable elegir la estructura de tres factores. Otro aspecto a tomar en cuenta es el porcentaje de varianza explicada. La estructura de tres factores encontrada en este estudio explica un menor porcentaje de varianza que la versión original del instru- mento que, como se ha dicho, incluye cuatro factores. Sin embargo, la diferencia entre las estructuras de tres y cuatro factores evaluadas en este estudio es mínima, pues ninguna supera el porcentaje mínimo recomendable de varianza explicada que de acuerdo con Pérez y Medrano (2010) es del 50\%. Por tanto, tomando los criterios de evidencia empírica y de varianza explicada, se considera pertinente seleccionar la estructura de tres factores.

La mayoría de los ítems quedaron ubicados en las dimensiones propuestas por los autores de la prueba excepto algunos que cargaron en factores distintos. En el factor motor cargaron los ítems 20 y 31 que en la estructura original pertenecían al componente fisiológico, así como los ítems 27 y 28, que pertenecían al componente cognitivo. En cuanto al ítem 20("Mis relaciones sexuales han disminuido"), su carga en el componente motor puede explicarse porque aunque tienen una implicación fisiológica en cuanto al deseo y satisfacción sexual, poseen también un componente motor relacionado con la ejecución y frecuencia con la que se llevan a cabo las relaciones sexuales. Respecto al ítem 31 ("Mi respiración se agita"), aunque es obvio que respirar es una función fisiológica tiene también una implicación motora, que aunque involuntaria, requiere un movimiento particular de los grupos musculares involucrados.

La carga de los ítems 27 ("Me cuesta mucho tomar decisiones") y 28 ("Tengo problemas para concentrarme y pensar con claridad") en el factor motor, podría explicarse porque la toma de decisiones tiene una implicación cognoscitiva en tanto requiere la reflexión acerca de las alternativas, sus pros y contras, pero podría tener una implicación motriz por la consiguiente ejecución de la decisión tomada. En lo que refiere a la dificultad para concentrarse y pensar con claridad, su implicación motriz podría guardar relación con el despliegue de comportamientos incompatibles con aquellos que facilitan la concentración como por ejemplo, dirigir la atención hacia estímulos distintos a aquellos en los que se debe enfocar la atención y concentración.

En el componente cognitivo cargó el ítem 21 ("Tengo dificultades para relajarme")que según los autores pertenece al componente fisiológico. Esto podría explicarse porque aunque la relajación tiene una dimensión fisiológica, también tiene que ver con la percepción de relajación no sólo física sino mental. El ítem 34 ("Lloro de forma incontrolada”), que según la teoría pertenece a la escala de ideación suicida, cargó en el componente fisiológico, lo que puede estar explicado porque la conducta de llorar tiene una implicación claramente fisiológica.

Por su parte, la exclusión de los ítems 11 ("En casa me encierro en mi habitación") y 24 ("Pienso que todo me va a salir mal"), podría estar explicada respectiva- 
mente por el hecho de que la muestra es en su mayoría de un estrato social medio-bajo y en este grupo social en Venezuela suele vivir en viviendas inadecuadas y en hacinamiento ocasionalmente crítico (Ponce, 2012), por lo que la posibilidad de encerrarse en su habitación podría ser prácticamente nula. Por otra parte, en lo que refiere al ítem 24, su exclusión podría explicarse por una de las características del venezolano, que presenta tendencia a ser optimista, lo que implica que tienda a tener expectativas positivas acerca del futuro (Rondón y Lugli, 2013). Por lo antes descrito, se propone emplear en población venezolana con enfermedades crónicas el instrumento conformado por 32 ítems que excluye los dos que no saturaron en ningún factor.

Los resultados del análisis de componentes principales, permiten observar que el instrumento presenta una estructura compuesta por tres factores (motor, cognitivo y fisiológico). Luego de evaluar con esta muestra la estructura de cuatro factores encontrada por los autores originales (motor, cognitivo, fisiológico y tendencia suicida), se observa que ésta no es la más adecuada, debido a los solapamientos en los componentes de los factores y la baja correspondencia de la estructura propuesta y la hallada en este análisis.

Considerando los aspectos antes descritos, aunados a que tal como se expresó en líneas anteriores, la estructura de tres factores fue la propuesta inicial de los autores del instrumento original, consonante con el modelo tridimensional que es la propuesta teórica en que se basa el instrumento (Martínez-Monteagudo et al., 2012), y teniendo en cuenta que el factor ideación suicida está compuesto por ítems que evalúan pensamientos asociados a la muerte (por lo que es esperable que se incluyan en el componente cognitivo), se sugiere mantener la estructura de tres factores para los fines del uso del instrumento en la población para la que ha sido validada.

El análisis factorial confirmatorio mostró índices de ajuste no satisfactorios en la estructura de tres factores, sin embargo, son más adecuados que los que se observaron tras el análisis factorial confirmatorio de la estructura de cuatro factores.

En lo que refiere a la validez de criterio, tras evaluar la relación de los puntajes obtenidos en el Cuestionario Tridimensional para la Depresión (CTD) y de cada uno de sus factores con las puntuaciones obtenidas por los participantes en la escala de ansiedad rasgo del Inventario de Ansiedad Rasgo-Estado de Spielberg (IDARE), se encontró una correlación alta y positiva entre la depresión y la ansiedad rasgo, lo que indica que a mayor tendencia a la ansiedad, mayor depresión, lo cual coincide con los hallazgos de autores que afirman que las personas que presentan mayor tendencia a responder de mane- ra ansiosa ante diversas situaciones, tienden más a estar deprimidos y a su vez tienden a presentar enfermedades (Moral, 2013; García y Calvanesse, 2008; Páez, Jofré, Azpiroz y De Bortoli, 2008; Roy-Byrne et al., 2008).

A partir de los resultados antes descritos, se considera que la validación venezolana del Cuestionario Tridimensional para la Depresión, debido a la óptima consistencia interna que presenta, es apta para ser empleada en estudios con pacientes que presentan enfermedades crónicas. Sin embargo, debido a que el modelo muestra ciertas debilidades psicométricas asociadas a un pobre ajuste de los datos, se sugiere realizar otras investigaciones con pacientes con otras enfermedades crónicas y distintas características sociodemográficas, de manera que sea posible aclarar la dimensionalidad de la escala y el ajuste del modelo propuesto para evaluar la depresión de manera tridimensional.

\section{Referencias}

Arechabala, M., Catoni, M., Palma, E., y Barrios, S. (2011). Depresión y autopercepción de la carga del cuidado en pacientes en hemodiálisis y sus cuidadores. Revista Panamericana de Salud Pública, 30 (1), 74-79.

Bharucha, A., y Wald, A. (2010). Trastornos anorrectales.Revista de Gastroenterología de México, 75, 497-507.

Bounchoucha, M., Devroede, G., \& Arsac, M. (2004). Anismus: a marker of multi-site functional disorders? International Journal of Colorectal Disease, 19, 374-379.

Cano-Vindel, A., Salguero, J., Wood, C., Dongil, E., y Latorre, J. (2012). La depresión en atención primaria: prevalencia, diagnóstico y tratamiento. Papeles del Psicólogo, 33 (1), 2-11.

Cogollo, Z., Díaz, C., y Campo, A. (2006). Exploración de la validez de constructo de la escala de Zung para depresión en adolescentes escolarizados. Colombia Médica, 37 (2), 102-106.

Edo, M., y Ballester, R. (2006). Estado emocional y conducta de enfermedad en pacientes con VIH/SIDA y enfermedades oncológicas. Revista de Psicopatología y Psicología Clínica, 11 (2), 79-90.

Fabián, M., García, M., y Cobo, C. (2010). Prevalencia de síntomas de ansiedad y depresión en pacientes con diabetes mellitus tipo 2 y su asociación con el tipo de tratamiento, complicaciones de la diabetes y comorbilidades. Medicina Interna de México, 26, 100-108.

Fernández, J., y Miralles, F. (2014). Estudio de la depresión en el asma bronquial a través del triple sistema de respuesta. Anuario de Psicología, 44 (1), 21-29.

Fernández, J. Simón, M., y Bueno, A. (2010). Depresión, género y manifestaciones sistémicas en las enfermedades inflamatorias crónicas del intestino. Psicothema, 22, 208-212.

Frasure-Smith, N., Lespérance, F., \& Talajic, M. (1995). The impact of negative emotions on prognosis following myocardial infarction: is it more than depression? Health Psychology, 14, 388-98.

Freire, M., Rodríguez, J., Möller, I., Valcárcel, A., Tornero, C., Díaz, G.,... Paredes, S. (2011). Prevalencia de síntomas de an- 
siedad y de depresión en pacientes con artritis psoriásica en consultas de reumatología. Reumatología Clínica, 7 (1), 20-26.

García, H., y Calvanesse, N. (2008). Calidad de vida percibida, depresión y ansiedad en pacientes con tratamiento sustitutivo de la función renal. Psicología y Salud,18 (1), 5-15.

Gil, B., Ballester, R., Gómez, S., y Abizanda, R. (2013). Afectación emocional de los pacientes ingresados en una unidad de cuidados intensivos. Revista de Psicopatología y Psicología Clínica, 18 (2), 129-138.

Gotlib, I., \& Joormann, J. (2010). Cognition and depression: current status and future directions. Annual Review of Clinical Psychology, 6, 285-312.

Gros, D., Anthony, M., McCabe, R., \& Swinson, R. (2009). Frequency and severity of the symptoms of irritable bowel syndrome across the anxiety disorders and depression. Journal of Anxiety Disorders, 23, 290-296.

Gutiérrez, J., y Portillo, C. (2013). La ansiedad y la depresión como indicadores de problemas de salud mental en los salvadoreños. Revista Electrónica de Psicología Iztacala, 16 (2), 533-557.

Hair, J., Anderson, R., Tatham, R., y Black, W. (2008). Análisis multivariante ( $5^{\mathrm{a}}$ ed.). Madrid, Prentice Hall.

Iwamitsu, Y., Shimoda, Z., Abe, H., Tani, T., Kodama, M., \& Okawa, M. (2003). Differences in emotional distress between breast tumor patients with emotional inhibition and those with emotional expression. Psychiatry and Clinical Neurosciences, 57, 289-294.

Jiménez, G., y Miguel-Tobal, J. (2003). El cuestionario tridimensional para la depresión (CTD): Un nuevo instrumento para medir la tristeza/depresión. Ansiedad y Estrés, 9 (1), 17-34.

Kerlinger, F., y Lee, H. (2002). Investigación del comportamiento. México, Mc Graw Hill.

Lang, R J. (1968). Fear reduction and fear behavior: Problems in treating a construct. En J.M. Shlien (Ed.), Research in psychotherapy (Vol. 3). Washington, DC, American Psychological Association.

Martínez-Monteagudo, M., Inglés, C., Cano-Vindel, A., y García-Fernández, J. (2012). Estado actual de la investigación sobre la teoría tridimensional de la ansiedad de Lang. Ansiedad y Estrés, 18 (2-3), 201-219.

Montoya, O. (2007). Aplicación del análisis factorial a la investigación de mercados. Caso de estudio. Scientia et Technica, 35.

Moral, J. (2013). Validación de un formato simplificado del inventario de depresión de Beck (BDI). Psicología Iberoamericana, 21 (1), 42-52.

Nogueda, M., Pérez, B., Barrientos, V., Robles, R., y Sierra, J. (2013). Escala de ansiedad y depresión hospitalaria (HADS): validación en pacientes mexicanos con infección por VIH. Psicología Iberoamericana, 21 (2), 29-37.

Oblitas, L. (2010). Psicología de la salud y calidad de vida (3ed.). México, CENGAGE Learning.

Oladeju, B., \& Gureje, O. (2013). The comorbidity between depression and diabetes. Complex Medical-Psychiatric Issues, 15, 390.

Páez, A., Jofré, M., Azpiroz, C., y De Bortoli, M. (2008). Ansiedad y depresión en pacientes con insuficiencia renal crónica en tratamiento de diálisis. Universitas Psychologica, 8, 117-124.

Pérez, E., y Medrano, L. (2010). Análisis factorial exploratorio: bases conceptuales y metodológicas. Revista Argentina de Ciencias del Comportamiento, 2 (1), 58-66.
Pescatori, M., Spyrou, M., \& Pulvirentid'Urso, A. (2006). A prospective evaluation of occult disorders in obstructed defecation using the 'iceberg diagram'. Colorectal Disease, 8, 785789.

Piqueras, J., Martínez, A., Ramos, V., Rivero, R., y García, L. (2006). Ansiedad, depresión y salud [Versión electrónica]. En L. Oblitas (Ed). Psicología de la Salud y enfermedades crónicas. Bogotá: PSICOM Editores.

Piqueras, J., Martínez, A., Ramos, V., Rivero, R., García, J., y Oblitas, L. (2008). Ansiedad, depresión y salud. Suma Psicológica, 15, 43-74.

Ponce, M. (2012). Los cambios de la pobreza en Venezuela a través del indicador de necesidades básicas insatisfechas. Censos nacionales de población y vivienda 1990-2011. Temas de Coyuntura, 66, 223-229.

Redondo, A., Calvanesse, N., García, H., y Hernández, L. (2010). Evaluación de factores psicológicos asociados a la nefropatía crónica del injerto. Argos, 27, 62-97.

Rondón, J., y Lugli, Z. (2013). Efectos del tipo de diabetes, estrategias de afrontamiento, sexo y optimismo en el apoyo social. Revista de Psicopatología y Psicología Clínica, 18, 193207.

Roy-Byrne, P., Davidson, K., Kessler, R., Asmundson, G., Psych, R., Goodwin, R., et. al (2008). Anxiety disorders and comorbid medical illness. General Hospital Psychiatry, 20, 208225.

Ruiz-Grosso, P., Loret, C., Vega-Dienstmaier, J., Arévalo, J., Chavez, K., Vilela, A., ..., Huapaya, J. (2012). Validation of the Spanish Center for Epidemiological Studies Depression and Zung self-rating depression scales: A comparative validation study. PLoS ONE, 7 (10).

Salguero, J., e Iruarrizaga, I. (2006). Relaciones entre inteligencia emocional percibida y emocionalidad negativa: Ansiedad, ira y tristeza/depresión. Ansiedad y Estrés, 12 (2-3), 207-221.

Sanz, J., y García-Vera, M. (2013). Rendimiento diagnóstico y estructura factorial del inventario de depresión de Beck-II (BDIII). Anales de Psicología, 29 (1), 66-75.

Spielberger, C.D., Gorsuch, R.L., y Lushene, R.E. (1982). Cuestionario de Ansiedad Estado/Rasgo. Madrid, TEA.

Stenman, M., Holzmann, M., \& Sartipy, U. (2014). Relation of major depression to survival after coronary artery bypass grafting. The American Journal of Cardiology, 114 (5), 698703.

Teva, I., Bermúdez, M., Hernández-Quero, J., y Buela-Casal, G. (2005). Evaluación de la depresión, ansiedad e ira en pacientes con VIH/SIDA. Salud Mental, 28, 40-49.

Trento, M., Raballo, M., Trevisan, M., Sicuro, J., Passera, P., Cirio, L., ... Porta, M. (2012). A cross-sectional survey of depression, anxiety and cognitive function in patients with type 2 diabetes. Acta Diabetológica, 49, 199-203.

Vázquez, C., Hernangómez, L., Hervás, G., y Nieto-Moreno, M. (2006). La evaluación de la depresión en adultos. En: V. Caballo (Ed), Manual para la evaluación clínica de los trastornos psicológicos. Trastornos de la edad adulta e informes psicológicos. (pp. 89-114). Madrid, Pirámide.

Wagner, F., González-Fortaleza, C., Sánchez-García, S., GarcíaPeña, C., y Gallo, J. (2012). Enfocando la depresión como problema de salud pública en México. Salud Mental, 35 (1), 3-11. 